\title{
AUTOPERCEPCIÓN COMO DOCENTES DE LOS BIBLIOTECARIOS DEL GRADO UNIVERSITARIO EN ENFERMERÍA EN ESPAÑA
}

\author{
César Manso-Perea* \\ Biblioteca. Colegio Oficial de Enfermería de Madrid. \\ Aurora Cuevas-Cerveró ${ }^{* *}$ \\ Facultad de Ciencias de la Documentación (UCM) \\ Eva García-Carpintero Blas *** \\ Escuela Universitaria de Enfermería - Fundación Jiménez Díaz (UAM) \\ Esther Martínez Miguel ${ }^{* * * *}$ \\ Facultad de Ciencias Biomédicas y de la Salud. Universidad Europea de Madrid.
}

\begin{abstract}
Resumen: Las funciones de los bibliotecarios en la universidad incluyen cada vez más responsabilidades relacionadas con la docencia. Se ilustra la relevancia del acceso y uso competente de la información en el Grado en Enfermería. El bibliotecario docente universitario emerge como la figura que debe asumir responsabilidad en la formación de los estudiantes en materia de información. El objetivo del estudio fue analizar las percepciones que estos tienen con respecto a su desarrollo profesional y sus funciones como docentes. Se optó por un estudio descriptivo e interpretativo con un abordaje cualitativo. Se realizaron 21 entrevistas semiestructuradas a bibliotecarios de 18 universidades españolas que imparten docencia en el Grado en Enfermería. Los hallazgos sugieren que los bibliotecarios demandan más formación pedagógica antes de comenzar su carrera bibliotecaria, colaboración de los docentes y reconocimiento por parte de la universidad para una perfecta comprensión de su concepto como nueva figura en la docencia.

Palabras clave: bibliotecario docente universitario; competencias informacionales; alfabetización informacional; bibliotecas universitarias; estudios de grado; enfermería.
\end{abstract}

Title: LIBRARIANS SELF-PERCEPTION AS TEACHERS OF THE NURSING DEGREE IN SPAIN.

Abstract: The functions of librarians in the university increasingly include responsibilities related to teaching. The relevance of the competent access and use of information in the Nursing Degree is shown. Academic librarian in University emerges as the accurate figure to assume responsibility in the "information management skills" student's training. The objective of this study was to analyze the librarians perceptions regarding professional development and their functions as teachers. Through qualitative approach, a descriptive and interpretive study was performed. Twentyone semi-structured interviews were conducted with librarians from 18 Spanish universities teaching in the Nursing Degree. The findings suggest that librarians demand more pedagogical training before starting their library career, mayor collaboration with teachers and institutional recognition as necessary for a perfect conceptual understanding of their new teaching figure.

Keywords: academic teaching librarian; information competences; information literacy; degree studies; nursing; university libraries.

Copyright: (C) 2021 Servicio de Publicaciones de la Universidad de Murcia (Spain). Este es un artículo de acceso abierto distribuido bajo los términos de la licencia Creative Commons Reconocimiento 4.0 Internacional (CC BY 4.0).

\section{INTRODUCCIÓN}

En el actual contexto tecnológico el crecimiento exponencial de la información exige un análisis constante de esta en cualquiera de sus formatos. La observación de este fenómeno junto a los cambios en el modelo educativo, orientado hacia un aprendizaje permanente o "a lo largo de la vida" (lifelong learning) convierten el acceso y uso de la información en competencias clave, que exigen conocimiento, habilidades y actitudes específicas. Esta necesidad es compartida por todos los niveles y entornos educativos y común a todas las disciplinas de estudio.

\footnotetext{
*biblioteca@codem.es

** macuevas@ccinf.ucm.es

*** eva.garciab@quironsalud.es

**** esther.martinez@universidadeuropea.es
}

Recibido: 22-09-2021; 2 $2^{\mathrm{a}}$ versión: 18-10-2021; aceptado: 19-10-2021.

MANSO PEREA, C.; CUEVAS CERVERÓ, A.; GARCÍA-CARPINTERO BLAS, E. y MARTÍNEZ MIGUEL, E. Autopercepción como docentes de los bibliotecarios del grado universitario en enfermería en España. Anales de Documentación, 2021, vol. $24, \mathrm{n}^{\circ} 2$. Disponible en: http://dx.doi.org/10.6018/analesdoc.492811. 
La transformación en los niveles educativos básicos es paulatina, y el desarrollo de estas competencias progresivo, en cualquier caso, por lo que en el momento de acceso a los estudios universitarios las habilidades para localizar, acceder, recuperar, evaluar y usar de forma crítica la información de forma autónoma no están del todo desarrolladas y/o no son suficientes. Surge entonces la necesidad de diseñar formaciones básicas para ese momento de transición educativa, específicas en el caso de algunas disciplinas como las Ciencias de la Salud, que permitan a los estudiantes interactuar con el proceso informacional de forma óptima. Esta formación debe favorecer el desarrollo en niveles de logro superiores de lo que los teóricos denominan competencias informacionales, digitales y multimedia, entre otras, y que son el objeto de la alfabetización informacional (ALFIN).

Sin embargo, a pesar de la importancia de las competencias informacionales y digitales en la educación universitaria, de la gran implicación y esfuerzo de REBIUN (en sus diferentes planes estratégicos), y del desarrollo del marco DigComp, promovido por la Unión Europea, la integración de estas formaciones no se ha producido de manera estratégica y planificada. Actualmente los proyectos para integrar la ALFIN en el diseño curricular de las titulaciones de Grado en Enfermería son desiguales, existiendo una tendencia al desarrollo no formal, a través de colaboraciones entre bibliotecario y docentes, promovidas por la motivación intrínseca y la actitud proactiva de unos y otros (Manso Perea, Cuevas Cerveró y González Cervantes, 2019).

Este tipo de colaboraciones son primordiales para el desarrollo de las competencias informacionales en los estudiantes, y el curso natural de las mismas conduce a la integración del bibliotecario como figura docente (Brassley, 2008; Wheeler y McKinney, 2015; González Fernández-Villavicencio et al., 2016). Formar al usuario potenciando su conocimiento y habilidades en este campo es una de las funciones principales de los bibliotecarios, que en el momento actual no solo ejercen de meros recopiladores del conocimiento o proveedores de servicios sino de facilitadores del propio conocimiento. Las enormes posibilidades tecnológicas actuales de gestión del conocimiento no sirven de nada sin las competencias necesarias para su localización, acceso, recuperación, evaluación y uso crítico. Quienes diseñan, implementan y gestionan a diario las herramientas que hacen posible todo esto son los bibliotecarios, por lo que restringir su papel formador y depositar la responsabilidad de la ALFIN en el equipo docente de la titulación es erigirles en una figura intermediaria que debe transmitir un conocimiento que no le es propio, mientras se desaprovecha un recurso específico y directo, el bibliotecario docente.

\section{REVISIÓN DE LA LITERATURA}

\subsection{Competencias informacionales en enfermería}

Los libros blancos elaborados con motivo del diseño de los títulos de grado en Ciencias de la Salud, y de forma específica en Enfermería, incluyen como competencia transversal la "gestión de la información". Para el desarrollo y logro de esta competencia a través de los planes de estudio se plantean varios aspectos clave.

El reconocimiento de las habilidades relacionadas con el manejo de la información, como elemento imprescindible para desenvolverse con éxito en la Sociedad de la Información y el Conocimiento, planteadas además como un proceso de aprendizaje a lo largo de la vida, ligado no solo al período formativo de grado sino también vinculado a habilidades de pensamiento crítico, solución de problemas y práctica de la medicina y la enfermería basadas en evidencia.

Las intervenciones enfermeras, tanto durante la formación universitaria como posteriormente en el desarrollo profesional de la práctica clínica, son intensivas en información, estando presentes en ella muchos elementos informacionales (Ortoll, 2004; García, 2010).

Los estudiantes deben enfrentarse por tanto desde fases muy tempranas a "nuevos retos pedagógicos, más orientados a la práctica, basados en la propia iniciativa y en la adquisición de destrezas para las búsquedas, el tratamiento y la organización de la información científica disponible" (Guerra et al., 2014).

Estos retos convierten en imprescindible un rol activo de los estudiantes en todas las actividades formativas, tanto en el aula como en el contexto del aprendizaje clínico, y para ello, necesitan conocimiento, habilidades y actitudes que les permitan localizar y gestionar la información de manera eficaz.

En este escenario, los estudiantes necesitarán además ser competentes en el uso de las TIC, para poder fortalecer su proceso de aprendizaje autónomo, clave en un entorno de complejidad y cambio tecnológico y científico permanente. Este aprendizaje se consolida con frecuencia en entornos virtuales de aprendizaje, lo que requiere individuos competentes en el uso y dominio de la información. "El profesional sanitario, tal como sucede en otros entornos, comienza su ejercicio profesional tras un periodo reglado de formación, y trabaja en un sistema que le provee de medios 
materiales para el desarrollo de su actividad. Sin embargo, existe una diferencia notable con otras profesiones en relación a sus necesidades de información, diferencia que puede centrarse en dos cuestiones: la repercusión que sus decisiones tienen sobre la salud de las personas y el acelerado ritmo de innovación científica en todas las facetas relacionadas con la salud" (García et al., 2011).

Otro aspecto que ilustra la relevancia del acceso y uso competente de la información en los profesionales enfermeros es la mejora progresiva de la calidad de los cuidados y la seguridad del paciente a través de la práctica basada en la evidencia. Así, la Enfermería Basada en la Evidencia (EBE) es un enfoque que reafirma la necesidad de las competencias informacionales como garantes de la búsqueda y acceso a la mejor evidencia científica disponible para guiar la toma de decisiones. Es importante también el conocimiento de aquellas herramientas que les permiten ser competentes en la gestión de la enorme producción científica sobre su disciplina.

Por último, la propia relación enfermera - paciente se ha visto transformada con la irrupción de las TIC y el desarrollo de la Sociedad de la Información y el Conocimiento. Las posibilidades de acceso a información en Ciencias de la Salud son cada vez mayores para los pacientes, pudiendo consultar sobre signos y síntomas, cuidados, fármacos, tratamientos alternativos, etc. y esto requiere de un profesional solvente en el acceso a la información, que conozca los recursos que puedan responder en cada momento a las necesidades de información del paciente, y que puedan hacerlo con garantías y desde la mejor evidencia científica disponible.

Utilizar los Trabajos Fin de Grado (TFG) o los trabajos de investigación en definitiva para integrar las competencias informacionales en el plan de estudios de la universidad, es otra oportunidad que debe ser aprovechada por los bibliotecarios para integrarse en la docencia (Bent y Stockdale, 2009; González Fernández Villavicencio et al., 2016).

La literatura sobre la integración de competencias informacionales en el currículo de enfermería por parte de bibliotecarios es abundante (Wallace et al., 1999; Dorner, Taylor y Hodson-Carlton, 2001; Jacobs, Rosenfeld y Haber, 2003; Tarrant, Dodgson y Law, 2008; Jacobsen y Andenaes, 2011; Bønløkke, Kobow y Kristensen, 2012; Janke, Pesut y Erbacker, 2012; Stombaugh et al., 2013; Özbiçakçı, Gezer y Bilik, 2015; Argüelles, 2016). En España son también varios los autores que describen algunas estrategias de integración en sus trabajos (García, 2010; Manso et al., 2015; Dominguez Aroca, 2017).

En cualquier caso, la literatura aboga, generalmente, por estrategias diseñadas en colaboración entre el profesorado de enfermería y los propios bibliotecarios.

\subsection{Bibliotecario formador. Bibliotecario docente. Bibliotecario docente universitario}

Las bibliotecas universitarias, generalmente, han ofertado dentro de su carta de servicios la formación de usuarios. Entendida esta como una formación relacionada con los servicios y recursos de la propia biblioteca. Esta actitud proactiva de los bibliotecarios no tenía la cuota suficiente de éxito en cuanto a la asistencia de usuarios en este tipo de formaciones. Muchas veces dependía del grado de implicación y recomendación de los docentes, recomendando estos cursos a los estudiantes como apoyo a sus materias. Además, este tipo de formaciones no tenían un "claro reconocimiento y apoyo institucional" (Hernández Hernández, 2010).

La transformación del concepto de formación de usuarios hasta alcanzar la formación en competencias informacionales o ALFIN, relacionada y solapada con otras alfabetizaciones; digital, mediática, multimedia, etc., supone una oportunidad irrechazable para el bibliotecario, que gradualmente va renunciando a la instrucción basada en habilidades en recursos o servicios de la biblioteca y se centra más en modelos de aprendizaje más holísticos y conceptuales. Un movimiento que aleja al bibliotecario como proveedor de servicios hacía un bibliotecario como profesor o compañero de enseñanza (Baer, 2021). El rol del bibliotecario que imparte formación relacionada con la ALFIN sería más visible y reconocido, si esta fuera planificada y estuviera integrada en la docencia (Manso et al., 2019).

En esta línea añade Gómez Hernández (2010) que actualmente "las bibliotecas universitarias españolas creen tener una función que va más allá del apoyo meramente instrumental a la actividad de enseñanza y aprendizaje. Una función "intrínsecamente" educativa, en la que se implican en profundidad y de la que han hecho una prioridad".

Peackock (2001) y Lupton (2002) (citados por Serrano Vicente, 2008, p. 510) afirmaron que, en las bibliotecas del siglo XXI, "los profesionales de la información se convertirían en bibliotecarios docentes (teacher librarians), y estarían en condiciones de equipar a los estudiantes universitarios con habilidades de búsqueda y evaluación de la información". Lupton describe la transición de "bibliotecario que enseña" a "bibliotecario profesor" afirmando que, si bien ambos 
enseñan ALFIN, el bibliotecario profesor lo hace desde una posición más consciente de su papel como parte integrante de un entorno educacional.

Peackock (2001) añade que los bibliotecarios deberían tener la consideración de docentes esgrimiendo que si la ALFIN forma parte de las competencias que se persiguen con el diseño curricular o plan de estudios de la titulación, debe ser impartida por especialistas, en este caso bibliotecarios. Lupton (2002) señala que los bibliotecarios deberían estar involucrados en el objetivo educacional de la universidad y participar activamente en los programas de ALFIN, trabajando en íntima colaboración con los docentes. Si la ALFIN debe ser impartida en profundidad e integrada en los diferentes planes de estudio de todas las disciplinas universitarias, debe hacerse con personal cualificado y el profesional cualificado para esta función es el bibliotecario.

Como hemos mencionado hasta ahora, la figura del bibliotecario docente emerge en la universidad y su función incluye cada vez más responsabilidades y habilidades relacionadas con la docencia. Incluso dedica más tiempo a la docencia que a otras tareas tradicionalmente más bibliotecarias (Overn, 2014). El bibliotecario docente universitario se va integrando en el modelo de enseñanza universitaria acompañando a los cambios y el desarrollo de los planes de estudio y las metodologías docentes con la formación de los estudiantes en competencias de gestión de la información, imprescindibles durante toda la formación, y especialmente relevantes en el desarrollo de los TFG (González Fernández Villavicencio et al., 2016). Los requerimientos de innovación docente en el contexto educativo actual redescubren como necesaria la alianza entre bibliotecario, docente y estudiante en los procesos de enseñanza-aprendizaje, lo que pone en evidencia el valor que aporta a la comunidad universitaria el bibliotecario docente.

Aunque bien es cierto que ni tan siquiera existe una denominación unívoca de esa figura. La bibliografía hace referencia a diversos conceptos como bibliotecario docente, bibliotecario formador, bibliotecario académico, etc. Esas denominaciones suelen estar relacionadas con el nivel educativo en el que se desarrollen. Descartaremos para la revisión bibliográfica el concepto de bibliotecario docente o su versión anglosajona teacher librarian, por confundirse, en ocasiones, con la figura del profesor que asume tareas bibliotecarias o por referirse a bibliotecarios docentes en niveles educativos anteriores a la universidad. Si bien utilizaremos el término bibliotecario académico universitario como bibliotecario que se integra en la docencia, en el plan de estudios de la universidad.

Ahora bien, definamos estos conceptos. Varios autores han trabajado las definiciones de estas figuras de bibliotecarios formadores, docentes, académicos (González-Fernández Villavicencio, 2016; Campal, 2019).

En España, un documento oficial, "Perfiles profesionales del Sistema Bibliotecario Español: fichas de caracterización" (Tejada y Martínez, 2019), recoge diferentes definiciones de formación de usuarios y ALFIN y de las figuras responsables de su impartición, pero no hace mención al bibliotecario docente universitario, formador que se integra en la docencia universitaria.

En el documento "Roles and Strengths of Teaching Librarians" (ACRL, 2017), como bien señala Campal (2019), se produce un cambio en la denominación, cambio de "competencias" a "roles" y de bibliotecario de "instrucción" (o formador) a "bibliotecario de enseñanza" (o docente). El propio documento "utiliza la denominación bibliotecario docente, definido como un bibliotecario que enseña en diversos contextos y para quien la enseñanza puede ser su responsabilidad profesional o parte importante de la misma. Esta acepción se usa porque se considera más amplia y más participativa que la de instrucción/formación, lo cual es un indicador de la importancia de la enseñanza y los objetivos educativos más amplios que tienen los bibliotecarios docentes en la actualidad" (Campal, 2019).

La literatura también nos ofrece ejemplos de colaboración de los bibliotecarios con los docentes (Brasley, 2008; Øvern, 2014; Mackey y Jacobson, 2005; Tiscareño y Cortes, 2014; Xu y Gil, 2017; Wishkoski, Lundstrom y Davis, 2018; Fundator y Maybee, 2019, p. 83) y escasamente información sobre el rol del bibliotecario integrado en la docencia universitaria. En este trabajo nos centraremos en la figura del bibliotecario docente universitario o el término utilizado en inglés academic teaching librarian; y más concretamente en el contexto de los estudios de Grado en Enfermería.

Podemos definir al bibliotecario docente universitario, academic teaching librarian, como el "bibliotecario de universidad que además de sus tareas habituales asume la impartición de clases a grupos de alumnos, profesores o investigadores a distinto nivel de responsabilidad, pero de forma regular, tanto presencial como virtualmente. Se trata de un profesional que planifica, diseña, imparte, evalúa y promociona las competencias de gestión de la información en su comunidad universitaria. La docencia forma parte de sus tareas habituales como bibliotecario" (González FernándezVillavicencio, 2016). 
Los bibliotecarios requieren otra denominación, que no les defina ya solo como tradicionales formadores de usuarios, sino en línea con la asunción de responsabilidades nuevas dentro de la formación, puesto que "ya no se enseña a usar una herramienta desde un punto de vista instrumental, se enseña a concretar el tema de la búsqueda; reflexionar sobre el sentido de lo que se va a buscar; definir la estrategia; presentar una metodología científica; desarrollar por escrito un trabajo científico; conocer y respetar los derechos de autor; presentar resultados de forma escrita, pero cada vez más de forma visual, mediante infografías y mapas mentales; defender en público unas ideas y resultados" (González Fernández-Villavicencio et al., 2016).

Hasta ahora se ha investigado poco sobre cómo los bibliotecarios perciben su labor como docentes, y nada, de forma específica, en la titulación de Grado en Enfermería. Existen trabajos previos que investigan sobre la identidad profesional de los bibliotecarios como docentes (Walter 2008; Julien y Pecoskie, 2009; Julien y Genuis, 2011; Austin y Bhandol, 2013) pero no existe mucha literatura, sobre cómo los propios bibliotecarios conciben sus habilidades de enseñanza o incluso si se consideran docentes. Trabajos como los de Wheeler y McKinney (2015) o más reciente de Baer (2021) son una excepción. Si bien en la investigación de Baer los bibliotecarios estaban comprometidos con su función docente, la de Wheeler y McKinney pretendía ofrecer una imagen más representativa de las diversas opiniones de los bibliotecarios sobre sus funciones docentes. Otro trabajo similar evalúa la autopercepción de los bibliotecarios docentes encargados de impartir la ALFIN en la universidad con respecto al liderazgo (Brooks, Warner y Hammons, 2021). Asimismo, el trabajo de Reale (2018), analiza la percepción de los bibliotecarios académicos: cómo se los ve y cómo se ven a sí mismos entre otros objetivos.

\section{OBJETIVOS}

Este estudio pretende ayudar a llenar este vacío explorando las percepciones de los bibliotecarios en relación a su desarrollo profesional y sus funciones como docentes en el contexto universitario. De manera específica, se trata de detectar necesidades de formación de la figura de bibliotecario docente, explorar su autopercepción acerca de su integración en el equipo docente, conocer las funciones desempeñadas por los bibliotecarios docentes y finalmente describir el diseño de las actividades académicas ALFIN integradas en el currículo de Grado en Enfermería.

\section{METODOLOGÍA}

\subsection{Diseño del estudio}

Para dar respuesta a los objetivos planteados, se optó por un estudio cualitativo descriptivo e interpretativo con una orientación teórico-metodológica basado en la fenomenología hermenéutica de Heidegger (Barberá e Inciarte, 2012). Desde esta perspectiva, se puede explorar e interpretar las experiencias y percepciones de las personas implicadas en el proceso.

\section{2 Ámbito y participantes del estudio}

Los participantes del estudio fueron los responsables de la formación en competencias informacionales de las mejores iniciativas de integración de competencias informacionales en el Grado en Enfermería en España. Para la selección de los entrevistados se utilizó la base de datos CIENF (Manso et al, 2019). Se optó por seleccionar a aquellos docentes con un perfil relacionado con las ciencias de la información y documentación.

Se realizó una recogida previa de datos sociodemográficos con el fin de caracterizar la muestra de docentes entrevistados y definir el perfil de bibliotecario docente. Estos datos incluían perfil profesional, experiencia bibliotecaria docente y una breve opinión personal sobre el tema objeto de estudio a modo de abordaje inicial de la entrevista (ver Tabla I). 


\begin{tabular}{|c|c|c|c|c|}
\hline Participantes & Sexo & Formación académica & $\begin{array}{l}\text { Formación en } \\
\text { docencia }\end{array}$ & CC.AA \\
\hline E1 & Mujer & Licenciada en Geografía e Historia & No & Madrid \\
\hline E2 & Mujer & Licenciada en Documentación & No & Cataluña \\
\hline E3 & Mujer & Licenciada en Documentación & No & Cataluña \\
\hline E4 & Mujer & Doctora en Documentación & No & Cataluña \\
\hline E5 & Mujer & Licenciada en Geografía e Historia & No & Galicia \\
\hline E6 & Mujer & $\begin{array}{l}\text { Licenciada en Historia / Máster en Información y } \\
\text { Documentación }\end{array}$ & No & Galicia \\
\hline E7 & Mujer & $\begin{array}{l}\text { Doctora en Filosofía / Máster en Bibliotecas } \\
\text { Digitales y Servicios de Información }\end{array}$ & $\begin{array}{l}\text { Sí, Curso de } \\
\text { Aptitud } \\
\text { Pedagógica }\end{array}$ & Madrid \\
\hline E8 & Mujer & Licenciada en Historia & No & Madrid \\
\hline E9 & Hombre & Licenciado en Documentación & Sí & Madrid \\
\hline E10 & Mujer & Licenciada en Documentación & No & Cataluña \\
\hline E11 & Mujer & $\begin{array}{l}\text { Diplomada en Enfermería / Licenciada en } \\
\text { Documentación / Doctora en Pedagogía }\end{array}$ & Sí & Cataluña \\
\hline E12 & Hombre & Doctor en enfermería & No & Cataluña \\
\hline E13 & Mujer & $\begin{array}{l}\text { Diplomada en Enfermería / Licenciada en } \\
\text { Documentación / Doctora en Documentación }\end{array}$ & No & Cataluña \\
\hline E14 & Hombre & Licenciado en Geografía e Historia & No & Galicia \\
\hline E15 & Mujer & Licenciada en Documentación & No & $\begin{array}{l}\text { Castilla y } \\
\text { León }\end{array}$ \\
\hline E16 & Mujer & $\begin{array}{l}\text { Licenciada en } \\
\text { Comunicación }\end{array}$ & Sí & $\begin{array}{l}\text { Castilla } \quad \mathrm{y} \\
\text { León }\end{array}$ \\
\hline E17 & Hombre & Licenciado en Documentación & No & Cantabria \\
\hline E18 & Mujer & $\begin{array}{l}\text { Licenciada en Geografía e Historia / Máster en } \\
\text { Bibliotecas }\end{array}$ & No & País Vasco \\
\hline E19 & Mujer & Licenciada en Geografía e Historia & No & Aragón \\
\hline E20 & Mujer & $\begin{array}{l}\text { Diplomada en Enfermería / Licenciada en } \\
\text { Documentación / Doctora en Documentación }\end{array}$ & $\begin{array}{l}\text { Sí, Curso de } \\
\text { Aptitud } \\
\text { Pedagógica }\end{array}$ & $\begin{array}{l}\text { Región de } \\
\text { Murcia }\end{array}$ \\
\hline E21 & Mujer & Licenciada en Historia & No & Andalucía \\
\hline
\end{tabular}

Tabla I. Datos sociodemográficos de la muestra. 


\begin{tabular}{|c|c|}
\hline $\begin{array}{ll}\text { ÁREA } & \text { DE } \\
\text { ESTUDIO } & \end{array}$ & PREGUNTAS PLANTEADAS \\
\hline $\begin{array}{l}\text { Formación } \\
\text { ALFIN de los } \\
\text { bibliotecarios }\end{array}$ & $\begin{array}{l}\text { 1 ¿Ha recibido formación continua/complementaria en competencias } \\
\text { informacionales? } \\
2 \text { Considera necesaria la preparación pedagógica de los bibliotecarios como nuevos } \\
\text { actores del panorama educativo. } \\
3 \text { ¿Debería estar presente en la titulación de Biblioteconomía y Documentación / } \\
\text { Documentación? } \\
4 \text { ¿Se siente suficientemente preparado para afrontar el reto? }\end{array}$ \\
\hline $\begin{array}{l}\text { Figura docente } \\
\text { de los } \\
\text { bibliotecarios. }\end{array}$ & $\begin{array}{l}1 \text { ¿Quién cree que debería impartir los contenidos en el aula? } \\
2 \text { Se considera reconocido, valorada su labor como docente } \\
3 \text { ¿Cómo se podría reconocer esta labor entre los bibliotecarios? } \\
4 \text { Considera que debería aparecer el nombre de la persona que imparte los contenidos } \\
\text { en competencias informacionales en la asignatura. } \\
5 \text { ¿Qué funciones como docente considera que debe asumir el bibliotecario docente? } \\
6 \text { ¿Considera que la formación en competencias informacionales mejora el perfil de } \\
\text { usuarios de biblioteca en los estudiantes? }\end{array}$ \\
\hline $\begin{array}{l}\text { Relación } \\
\text { bibliotecario- } \\
\text { Docente / } \\
\text { Docente- } \\
\text { Bibliotecario }\end{array}$ & $\begin{array}{l}1 \text { ¿Cómo definiría la relación bibliotecarios y docentes en las asignaturas que } \\
\text { imparte y en general? } \\
2 \text { Considera imprescindible la colaboración entre bibliotecarios y docentes para el } \\
\text { diseño de actividades académicas, ¿de qué forma? }\end{array}$ \\
\hline $\begin{array}{l}\text { Limitaciones } \\
\text { encontradas }\end{array}$ & $\begin{array}{l}1 \text { Principales obstáculos con los que se ha encontrado para implementar contenidos } \\
\text { relacionados con las competencias informacionales en los estudios de Grado en } \\
\text { Enfermería. } \\
2 \text { ¿Cómo consigue transmitir a los estudiantes la importancia de la adquisición de } \\
\text { competencias informacionales para su desarrollo como estudiante y para su futuro } \\
\text { profesional? }\end{array}$ \\
\hline
\end{tabular}

Tabla II. Guion de preguntas entrevista.

Se alcanzó la saturación de datos en el 21 participante, siendo todos bibliotecarios docentes de 18 universidades españolas que imparten clase en la titulación de Grado en Enfermería.

\subsection{Instrumentos de recogida de información}

En la recogida de datos se utilizó la entrevista semiestructurada realizada individualmente, cara a cara con el entrevistado. En base a la revisión de la literatura y la información proporcionada por la base de datos CIENF, y en combinación con las preguntas de investigación derivadas de la experiencia profesional del investigador principal, se diseñó en la segunda fase del estudio un guion de entrevista semiestructurada que recogía las áreas temáticas de interés en relación con el objeto de estudio (ver Tabla II).

Aunque las preguntas se formularon en un orden específico, siguiendo la metodología pertinente de entrevistas semiestructuradas, los distintos participantes fueron respondiendo libremente ampliando las respuestas y preguntas 
cuando el entrevistador lo considero. Fueron conducidas de forma dinámica a través de las preguntas del mencionado guion, transformando posteriormente los datos en categorías y unidades de significado para su posterior análisis.

Las entrevistas fueron grabadas en su totalidad en audio, con el consentimiento escrito del entrevistado, garantizando su confidencialidad y la Ley de Protección de Datos y Garantías Digitales. Conjuntamente los audios fueron completados con sus correspondientes notas de campo de todas aquellas observaciones y lenguaje no verbal relevante durante el desarrollo de dichas entrevistas.

\subsection{Análisis de los datos}

El proceso y análisis de datos realizado en el presente trabajo abarca la recogida de datos, transcripción, reducción, exposición, interpretación y finalmente verificación de los mismos, y de sus significados emergentes y fue concluida en 2017.

El proceso de reducción de datos se realizó en dos fases: separación de unidades de significado e identificación y clasificación de unidades. Las unidades de significado se consiguieron, una vez transcritas las entrevistas y a partir de una lectura de estas, reflexionando sobre su contenido y perfilando las relaciones que pudieran establecerse en ellas. A esta lectura se sumó la revisión de la literatura previa y la propia reflexión del investigador sobre la cuestión objeto de estudio. Posteriormente se procedió a identificar y clasificar las unidades mediante la codificación y categorización. Se decidió utilizar un proceso de categorización mixto (deductivo-inductivo). Es decir, por un lado, a partir de un mapa de familias y categorías establecido previamente por el investigador, se diseñaron las unidades de significado, y a continuación se realizaron cambios que posibilitaron la adaptación al conjunto de datos al que fueron aplicadas. A lo largo de todo el proceso de categorización y codificación de los significados que surgieron en las narraciones de las entrevistas, aparecieron algunos datos que podemos considerar inductivos, los cuales se interrelacionaron con los deductivos.

En la exposición de los datos, para garantizar el anonimato de los entrevistados y para agilizar la localización de citas textuales de estos se utilizó un sistema de codificación alfanumérico para identificar a cada uno de los participantes. Los códigos se construyeron utilizando los siguientes caracteres: letra E seguida de un número correlativo desde el 1 hasta el número total de participantes.

Para la interpretación de datos se utilizaron las conclusiones de los resultados obtenidos en las entrevistas junto con las notas de campo recogidas. Finalmente se consultaron algunos datos y las propias notas de campo con los entrevistados para evaluar su veracidad. Para importar la transcripción de cada entrevista y apoyar el análisis de estas, se utilizó el software ATLAS.ti 6.0.

Para garantizar la calidad del estudio, se siguieron las recomendaciones de los Consolidated Criteria for Reporting Qualitative Research COREQ (Tong, Sainsbury y Craig, 2007). Además, se emplearon criterios de rigor y calidad científica de los estudios cualitativos propuestos por Guba y Lincoln (Carpenter y Suto, 2008, p. 162); Pope y Mays, 2019, p. 142).

\section{RESULTADOS}

A continuación, se presentan los resultados más relevantes que se han obtenido en relación a la familia bibliotecario docente y sus dos categorías, formación académica e integración y reconocimiento en el equipo docente. En la categoría formación académica del bibliotecario docente se recogen las opiniones de los docentes entrevistados sobre si han recibido formación en ALFIN y si es necesaria la preparación pedagógica de los bibliotecarios como nuevos actores del entorno educativo. A lo largo de los resultados se recogen algunas de las citas más significativas sobre las percepciones de los entrevistados. Previamente cada docente describe su perfil profesional. Una unidad de significado emerge de forma dominante en las opiniones de los entrevistados, "Necesidad de formación pedagógica", que hace referencia a la necesidad de capacitar en competencias pedagógicas a los bibliotecarios para actuar de forma directa, activa y continuada en el proceso formativo de los estudiantes.

\section{Categoría: formación académica en el bibliotecario docente}

\section{Trayectoria formativa de los docentes en ALFIN}

Los docentes exponen si han recibido algún tipo de formación continua o complementaria a su título sobre ALFIN. La mayoría no ha recibido formación de este tipo, son autodidactas, pero sí refieren haber recibido formación en recursos o herramientas facilitadoras para la ALFIN.

"La verdad es que curso especifico yo creo que no, esto es autodidacta”. E14 
"Pues poca, yo creo, más bien poca nada. Todo es aquí, por nuestra cuenta. A lo mejor en algún congreso que te dicen algo. Pero yo no”. E8

\section{Necesidad de formación pedagógica}

Consideran muy necesaria la preparación pedagógica de los bibliotecarios para impartir estos contenidos en el aula. Por formación pedagógica entienden cuestiones relacionadas con liderazgo en aula, dicción, gestión de grupos, diseño de actividades formativas y manejo de herramientas docentes, entre otras.

"Totalmente, imprescindible. Preparación pedagógica, me hubiera encantado haberla tenido. A base de horas de formación se te va quitando un poco ese miedo". E1

"Es fundamental. El primer año lo pasas mal, porque, además, de tener la mentalidad en formación continuada con profesionales, el lenguaje, la estructura, los tiempos... y a mi me costó adaptarme. Por lo tanto, considero necesaria que haya una preparación pedagógica, cursos... si. La universidad los tiene, yo también he ido de liderazgo, de aplicación de nuevas tecnologías, etc.”. E6

Por otro lado, también opinaban sobre la necesidad de integrar este tipo de formación pedagógica en la titulación de Grado en Documentación. Casi la totalidad de los docentes respondió afirmativamente.

"A ver, yo creo que desde el momento que somos CRAI y que tenemos un EEES que tiene unas pautas; la figura del bibliotecario formador es básica y fundamental, igual que bibliotecario catalogador, o sea a este nivel. Igual que hicimos dos años de catalogación y tal, o sea, esta figura es básica y fundamental. El otro día estuve repasando las asignaturas de la universidad de lo nuestro, y no hay ninguna asignatura especifica de cómo formarte, y me parece.... Porque es que yo pondría una asignatura cada año, es básico [...]". E3

\section{Categoría: Integración y reconocimiento en el equipo docente}

Se recoge lo expresado por los bibliotecarios con respecto a su incorporación al equipo docente y su implicación en el proceso enseñanza-aprendizaje haciendo mención de las funciones que deben tener y la percepción que, sobre su nuevo rol y su compromiso en el espacio curricular, tienen los estudiantes, la universidad y ellos mismos.

Se han recogido 5 unidades de significado, destacando el porcentaje de frecuencia de la categoría Visión de los docentes.

\section{Visión de los estudiantes}

En esta unidad de significado, los entrevistados exponen las percepciones que tienen los estudiantes sobre su rol de docente. Para ellos, los estudiantes generalmente tienen una imagen positiva de los bibliotecarios docentes.

"Yo creo que lo perciben bien, las encuestas que hacemos pienso que lo perciben bien. Incluso alguno nos llama profesor, aunque luego nos vean en la biblioteca". E14

Los estudiantes valoran al docente positivamente, especialmente si los contenidos que imparten son percibidos como útiles para su vida académica.

"Todo depende de lo que tengan que hacer después con lo que yo les estoy enseñando. Si yo lo que les estoy enseñando no les va a servir para nada, es como que les resbala todo totalmente. Pero si ellos luego van a tener que hacer un trabajo, van a tener que hacer una evaluación, van a tener que hacer una bibliografia, van a tener que hacer una búsqueda bibliográfica; entonces sí”. E8

Los docentes también refieren el reconocimiento de los estudiantes con respecto a la labor que ellos realizan:

"Bien, son super receptivos, son super agradecidos, después de la clase te solicitan ayuda con un por favor, por favor y gracias y mil gracias". E3

Algunos docentes consideran importante crear un buen clima en el aula para poder ganarse al grupo y despertar interés por la asignatura, algo complicado al no ser una asignatura de contenido clínico.

"Yo lo que intento es crear cercanía con ellos [...] también me interesa luego los veré en la biblioteca, son mis clientes". E15

\section{Visión de la universidad}

Esta unidad de significado surge de la reflexión de los docentes sobre la necesidad de que aparezca el nombre de la persona que imparte los contenidos o el nombre de biblioteca en la guía docente, como un indicador de reconocimiento del centro o de la universidad a la labor docente de los bibliotecarios. Solo 12 docentes de los 21 entrevistados ven su nombre incluido en la guía docente. La mayoría de los bibliotecarios docentes lo considera necesario, algunos lo consideran importante por respeto a los estudiantes, al considerar la guía docente un contrato que se establece con ellos, $\mathrm{y}$ varios refieren una baja exigencia en relación a este aspecto concreto.

"En mi caso aparece, porque yo soy la responsable de la asignatura, con lo cual, evidentemente sí. Aparece y debería de aparecer". E6 
"Hombre, si formas parte de una asignatura y hay unas sesiones que son a tu cargo, tienes la responsabilidad de la evaluación, de la gestión de la clase, debería aparecer. Pero no porque sea más importante o menos, es una cuestión de respeto también al estudiante que tiene que saber quién le está hablando”. E3

También atribuyen su importancia a la necesidad de que haya una bolsa laboral con profesionales competentes para formar en este tipo de contenidos:

"[...] si que debe aparecer, si imparto contenidos como otro profesor [...] porque claro el currículum también es importante, yo siempre lo digo, a veces que solicitan formadores y tal, es importante el currículum, porque no todo el mundo sabe formar. Debes tener experiencia acreditada, no, si una persona tiene experiencia acreditada y puede acreditarlo, pues si'”. E3

\section{Visión de los docentes}

Por su parte esta unidad de significado surge de los sentimientos de los profesionales de la información y la documentación sobre su preparación para enfrentarse al reto de ser docentes y sobre el reconocimiento de su labor, y en caso de percibir que este reconocimiento no existe, cómo sienten que se les podría reconocer. La mayoría de los entrevistados considera estar preparados para afrontar el reto, demandando más formación de formadores y formación pedagógica específica, como se ha visto en una categoría anterior:

"Yo me siento preparada, sobre todo en cuanto ya tienes una amplia experiencia, pero es por pura experiencia, no porque yo haya recibido ninguna preparación, sino por experiencia. Pero sí que demandaría más formación en ese aspecto pedagógico, ¿no? Es decir, un poco más como tratar a los alumnos y como prepararlos mejor”. E1

También encuentran otros posibles obstáculos a pesar de sentirse suficientemente preparados para afrontarlo, como la falta de tiempo, puesto que los bibliotecarios además de la formación tienen que continuar con sus funciones tradicionales.

"[...] yo creo que actualmente de todos los trabajos que se hacen dentro de un CRAI, para mí, es el mejor con diferencia, el más gratificante, el más creativo, el más productivo, el que tiene más vínculos con la universidad y con la formación integral de los universitarios. Para mí es un trabajo que me gusta mucho, lo que pasa es que bueno, son las horas, trabajas las horas que trabajas, tienes tu vida propia y no puedes". E3

En cuanto al reconocimiento, ya sea en cuanto a satisfacción personal, reconocimiento económico o de acreditación, hay más discrepancias. Las diferencias, en ocasiones, se manifiestan en función del tipo de contrato que tiene la persona que imparte esos contenidos. La mayor satisfacción en este sentido es asociada muchas veces al reconocimiento que realiza de forma directa el propio estudiante:

"Satisfacción personal toda. Reconocimiento institucional como docentes, ninguno. Ahora yo desde luego a nivel personal toda, porque cuando tú ves que los alumnos aprenden las herramientas, aprenden las competencias; para mí desde luego toda la satisfacción es personal”. E21

En cuanto al reconocimiento en forma de remuneración o acreditación, salvo los contratados como profesores asociados, la mayoría no se considera satisfecho.

"No, de hecho, veo que la escasez de profesores en estas asignaturas es precisamente lo poco reconocido. Por parte de tu centro de trabajo, que no te permiten, como a un médico, ¿por qué yo tengo que pedir permiso para ir a dar clase a una asignatura cuando este es un centro asociado a la universidad, y un médico no tiene que pedir permiso? Si él asume que es profesor asociado y ya, yo soy profesora asociada igual, por qué tengo que pedir permiso a la dirección. Entonces muy mal reconocido, ni desde el punto de vista económico ni desde el punto de vista profesional”. E1

"En mi caso remuneración ninguna. Acreditación tampoco, porque aquí no vale para concurso de traslado, te cuenta cuando lo has recibido, pero no cuando lo has dado. Nos conformamos con que lo piden año tras año y será porque la gente está satisfecha con lo que haces". E15

Algunos docentes si se consideran reconocidos, puesto que están contratados como profesor asociado:

"Mi trabajo como docente si que me resulta satisfactorio, el reconocimiento profesional por parte de compañeros lo tengo y me siento muy bien valorada. La remuneración económica, bueno pues yo ahora mismo me siento bien y me parece que sí que estoy reconocida a través de la figura del asociado [...] Mira, dentro de la universidad hay como a tiempo completo y a tiempo parcial. A tiempo parcial, es el asociado; que es una figura experta en su campo y que la contratan a tiempo parcial para impartir un poco de acuerdo con la experiencia que tiene". E6

En cuanto a las posibles soluciones, los docentes proponen la remuneración económica, acreditación o la creación de un perfil de bibliotecario docente:

"Hay una opción que podría ser reconocimiento a través de certificación; como reconocimiento de horas lectivas, a mí se me ocurre que puede ser. De hecho, los tutores de prácticas que tenemos en los hospitales, en 
nuestro caso en la facultad no son asociados a nuestra facultad, son tutores. Ellos tienen un reconocimiento certificado, tantas horas, tantos créditos, el equivalente. Y es una certificación que, a nivel curricular, ahí está. Que como mínimo yo considero que debería de ser". E20

"Como mínimo deberían de estar remunerados como algo diferente, como mínimo, o sea eso es porque es un trabajo adicional, no es un trabajo. Porque si el jefe de recursos humanos da clase de derecho laboral en la universidad, no lo da gratis. Entonces el bibliotecario tampoco tiene porque darlo gratis, esa es la cuestión". E7

"Sería interesante que se hicieran perfiles, un perfil de bibliotecario docente, eso, por ejemplo. Que se tuviese en cuenta a la hora de los concursos, de méritos, de personal”. E8

\section{Funciones del bibliotecario docente}

Esta unidad de significado viene condicionada por el propio carácter y diseño de la asignatura; si es una asignatura específica, si está embebida en otra o si es un curso paralelo al currículo formal. Los entrevistados manifiestan las funciones que deben asumir como docentes:

"Sí hago todo. Todo, los materiales, los bancos de preguntas, tutorías, evaluación, las encuestas, el calendario, organización y tal”. E10

“Yo en mi caso, de cara a la docencia, hago todas. Evaluación y tutorías pre y post exámenes”. E6

Algunos entrevistados consideran innegociable la función de evaluar:

“A mí me gusta que cada uno pueda evaluar lo que ha dado porque tú sabes mejor lo que le puedes exigir a ese alumno. Es muy raro que yo de la asignatura y luego la evalúe otro, eso es muy raro; [...]. Yo creo que cada uno tiene que evaluar lo que da". E13

"Si, de hecho, creo que es importante, porque si es una materia que se imparte y no se evalúa; no tiene sentido, porque ya le estás tú mismo diciendo al alumno que no es evaluable. Y el alumno entiendo, con rol de alumno, que lo no evaluable no es importante. [...] Por supuesto que tiene que tener tutoría y que tiene que tener un trabajo evaluable”. E20

\section{Diseño actividades académicas}

Finalmente, esta unidad de significado surge de la visión que tienen los entrevistados de la relación con el resto de profesores del grado y de la consideración o no como necesaria de la colaboración mutua para el diseño de actividades académicas. La colaboración con el resto de los docentes como un elemento clave del proceso enseñanza aprendizaje está presente en el discurso de todos, $\mathrm{y}$, sin embargo, la existencia de colaboración es muy heterogénea.

"[...] si nosotros colaboramos Y ellos saben perfectamente la materia que se da en la asignatura de $1^{\circ}$ y hacen alusión a ella cuando le piden los trabajos, etc. Evidentemente le solicitan que busquen en bases de datos cientificas tales como las que se han explicado en $1^{\circ}$, la bibliografia tiene que estar normalizada según la normativa que se ha explicado en $1^{\circ}$ [...]”. E20

"La colaboración con docentes es condición sine qua non. Que sea una asignatura en un curso, que sea reglada y tal. [...] Y que además todos los profesores conocieran lo que se lleva a cabo y tuvieran que reconducir a sus alumnos a que utilizaran esos recursos". E19

Esta colaboración, en ocasiones, resulta escasa y los bibliotecarios docentes entrevistados demandan participar más en la planificación y organización general del diseño curricular, junto al resto de profesores. Colaboración en el planteamiento de los objetivos de aprendizaje, adaptación de guías docentes, defínición de contenidos, diseño de actividades o en los métodos de evaluación. Principalmente destacan la necesidad de que los profesores exijan al estudiante el dominio de los contenidos trabajados para adquirir las competencias informacionales y su aplicación para el logro de los objetivos de aprendizaje del resto de asignaturas.

"Es decir, yo te lo encarrilo a la ALFIN pero tú como profesor le vas a pedir al menos una actividad que tengan que utilizar la competencia informacional”. E11

"[...] y aparte lo que es importante es la coordinación con el resto del profesorado, y crear la necesidad. Que los profesores creen necesidades de información en los estudiantes”. E4

\section{DISCUSIÓN}

La formación en competencias de gestión de la información ha impulsado enormemente el perfil de bibliotecario académico universitario, asumiendo estos una carga lectiva creciente, y teniendo que superar las barreras de falta de experiencia, reconocimiento y valoración por parte de otros docentes y de la propia Administración. Este desarrollo debería acompañarse, como sucede en otros países, de un reconocimiento y certificación de la que aún no gozan en España (González Fernández-Villavicencio, 2016). 
La incorporación de los bibliotecarios al proceso de enseñanza-aprendizaje como una figura más del panorama educativo requiere del profesional la mejora de sus conocimientos, habilidades y actitudes. Para formar a los usuarios en habilidades de información, o bien para desarrollar productos o servicios dirigidos a este objetivo, los propios bibliotecarios deben estar a su vez alfabetizados.

A la vista de los resultados se puede deducir que los bibliotecarios entrevistados demandan una preparación pedagógica que no ha estado lo suficientemente presente en las titulaciones de Biblioteconomía y Documentación. Esta preparación sí que ha estado presente en las titulaciones en otros países (Bewick y Corrall, 2010; Simmons y Corrall 2011). En concordancia con los trabajos de Westbrock y Fabian (2010); Bewick y Corrall (2010); Austin y Bhandol, (2013); Wheeler y McKinney (2015) y Ducas, Michaud-Oystryk y Speare (2020). Además, añaden que adquieren la mayoría de las habilidades de enseñanza en el trabajo o de forma autodidacta, aunque habrían preferido aprender más de ellas antes de comenzar su carrera bibliotecaria de lo que realmente hicieron.

Incluso cuando algunos de los entrevistados consideran propia e individual la responsabilidad de aprender a enseñar, consideran que desde las escuelas de biblioteconomía o las instituciones educativas empleadoras se debería hacer más para apoyar a los bibliotecarios en el desarrollo de sus competencias en este sentido.

A través de sus respuestas, la gran mayoría de los participantes confirmaron que su trabajo de docentes había cambiado la percepción de los estudiantes con respecto a sus funciones dentro de la universidad, a pesar de que para los estudiantes es algo imprevisto, singular, ya que siempre han considerado a la biblioteca y a los bibliotecarios como un soporte, como un proveedor de servicios.

Si la formación de los bibliotecarios se imparte a estudiantes que tienen un trabajo de investigación designado, encuentran un aliciente, aumentando el nivel de participación y, en consecuencia, obteniendo mejores resultados (Bent y Stockdale, 2009; Limberg, Alexandersson y Lantz, 2008, Gonzalez Fernández-Villavicencio, 2016).

Por otro lado, los entrevistados refieren que los estudiantes que han recibido formación en ALFIN por parte de bibliotecarios se transforman en usuarios que hacen un mayor y mejor uso de la biblioteca y sus recursos.

El reconocimiento de la universidad hacia los bibliotecarios en las labores docentes ha sido mínimo, porque el propio sistema educativo no ha sabido integrar la biblioteca en este proceso. En muchas ocasiones el trabajo del bibliotecario académico universitario es equiparable al de un profesor asociado en su misma universidad, pero no recibe el estatus de docente, salvo que lo haga en otra universidad, o en postgrados, no observándose diferencias entre ambos perfiles profesionales (González Fernández-Villavicencio y otros, 2016; Sobel, Ramsey y Jones, 2018).

Incorporarse al rol docente es para los bibliotecarios un proceso complejo, que implica transformación profesional, de rol y de modelo. Se trata de "invadir" una tarea tradicionalmente desempeñada por otros, no demandada explícitamente y en un contexto competitivo, ya que dichos cambios tienen implicaciones en las esferas económica y organizativa. Sin embargo, y a pesar de lo descrito, esto no debería resultar traumático para una profesión que vive en constante cambio y transformación, y que se ha reinventado otras veces.

Los participantes expresaron con frecuencia un cambio hacia un aprendizaje más activo; la colaboración con otros educadores y a veces con los estudiantes; y una comprensión más profunda de la pedagogía que a menudo coincidía con un enfoque más holístico e integrado de la enseñanza y el aprendizaje. Estos cambios de enfoque suelen ir acompañados de una mayor confianza en los propios conocimientos pedagógicos y de un deseo y una capacidad de ser más flexible y experimental en la enseñanza.

A pesar de que muchos de los participantes manifestaron su entusiasmo por la docencia, entendida como el proceso de enseñanza y aprendizaje, también expresaron frustración por los obstáculos encontrados, que impiden un aprendizaje más profundo de los estudiantes y merman las oportunidades de los bibliotecarios para guiar y acompañar ese aprendizaje. Entrevistas de investigación más profundas como las de Walter (2008), Julien y Pecoskie (2009), Austin y Bhandol (2013) sugieren que tales frustraciones son comunes. Cada participante de este estudio parte de su propio contexto individual y único y sus propias experiencias en relación con la enseñanza y el aprendizaje, pero también de experiencias y puntos de vista compartidos sobre su trabajo y su labor pedagógica. Todos coinciden en que han tenido que desarrollar muy rápido competencias para impartir docencia relacionada con la ALFIN, pero no perciben su capacidad de liderazgo para avanzar en la enseñanza universitaria en materia de información asumiendo habilidades en el aula como las de comunicación, creación de equipos, asunción de riesgos o de confianza en sí mismo (Brooks, Warner y Hammons, 2021). 


\section{CONCLUSIONES}

Los bibliotecarios académicos universitarios muestran confianza y se sienten preparados y predispuestos como nuevos agentes en la docencia, participando en el desarrollo de competencias de gestión de la información. Perciben como necesaria la inclusión de formación en competencias pedagógicas en los grados en Documentación, que les capacite, entre otras cosas, para diseñar actividades formativas, elaborar materiales docentes, ejercer liderazgo en el aula, gestionar las nuevas tecnologías, diseñar programas educativos o de integración de equipos multidisciplinares.

La ausencia de esta formación tiene un impacto directo no solo en las competencias pedagógicas de los bibliotecarios, sino también, en su autopercepción como docentes, siendo en ocasiones fuente de inseguridad y alterando la percepción de autonomía en el rol docente. Esta autonomía, cuando existe y es percibida, no se acompaña del reconocimiento de sus funciones como docentes, existiendo límites confusos sobre la responsabilidad de la formación en ALFIN en el contexto universitario, escasas y heterogéneas interacciones con otros docentes y un apoyo institucional débil.

El nuevo rol docente retorna en un mayor y mejor uso de la biblioteca por parte de los estudiantes, quienes valoran positivamente cualquier actividad académica relacionada con la gestión de la información, si está vinculada a un trabajo académico (TFG). Que estas actividades sean diseñadas y lideradas por el bibliotecario se valora como especialmente positivo, ya que este es vinculado a la biblioteca, unidad de información por excelencia.

Si bien este estudio permite conocer las percepciones de una amplia población de bibliotecarios que imparten docencia, existen algunas limitaciones. La selección de la muestra se hizo buscando las mejores iniciativas de integración de competencias informacionales en el Grado de Enfermería, por lo que los bibliotecarios entrevistados parten de un contexto individual e institucional que puede favorecer la identificación con el nuevo rol docente. Futuras investigaciones podrían orientarse a evaluar el impacto de la actividad docente en ALFIN por parte de los bibliotecarios en los resultados de aprendizaje de los estudiantes.

\section{AGRADECIMIENTOS}

Los autores agradecen al grupo de bibliotecarios, docentes y bibliotecarios docentes universitarios que participaron en esta investigación. Queremos agradecer a los evaluadores del artículo por sus aportes y comentarios.

\section{BIBLIOGRAFÍA}

ACRL. Roles and Strengths of teaching Librarians. College \& Research Libraries News, 2017, vol. 78, $\mathrm{n}^{\circ} 7$, p. 364. Disponible en: https://doi.org/10.5860/crln.78.7.364.

ARGÜELLES, C. Curriculum-integrated information literacy (CIIL) in a community college nursing program: a practical model. Community College Journal of Research and Practice, 2016, vol. 40, $\mathrm{n}^{\circ}$ 11, p. 942-953. Disponible en: https://doi.org/10.1080/10668926.2016.1147395.

AUSTIN, T. y BHANDOL, J. The academic librarian: Buying into, playing out, and resisting the teacher role in higher education. New Review of Academic Librarianship, 2013, vol. 19, $\mathrm{n}^{\circ}$ 1, p. 15-35. Disponible en: https://doi.org/10.1080/13614533.2012.740438.

BAER, A. Academic librarians' development as teachers: A survey on changes in pedagogical roles, approaches, and perspectives. Journal of Information Literacy, 2021, vol. 15, $\mathrm{n}^{\mathrm{o}}$ 1, p. 26-53. Disponible en: https://doi.org/10.11645/15.1.2846.

BARBERA, N. e INCIARTE, A. Fenomenología y hermenéutica: dos perspectivas para estudiar las ciencias sociales y humanas. Multiciencias, 2012, vol. 12, n 2, p. 199-205.

BENT, M. y STOCKDALE, E. Integrating information literacy as a habit of learning -assessing the impact of a golden thread of IL in the curriculum. Journal of Information Literacy, 2009, vol. 3, $\mathrm{n}^{\mathrm{o}}$ 1, p. 43-50. Disponible en: https://doi.org/10.11645/3.1.212.

BEWICK, L. y CORRALL, S. Developing librarians as teachers: a study of their pedagogical knowledge. Journal of Librarianship and Information Science, 2010, vol. 42, $\mathrm{n}^{\mathrm{o}}$ 2, p. 97-110. Disponible en: https://doi.org/10.1177/0961000610361419.

BØNLØKKE, M.; KOBOW, E. y KRISTENSEN, A.K. Curriculum Integrated Information Literacy-a Challenge. Nordic Journal of Nursing Research, 2012, vol. 32, $\mathrm{n}^{\mathrm{o}}$ 3, p. 53-55. Disponible en: https://doi.org/10.1177/010740831203200311.

BRASLEY, S.S. Effective librarian and discipline faculty collaboration models for integrating information literacy into the fabric of an academic institution. New Directions for Teaching and Learning, 2008, $\mathrm{n}^{\circ} 114, \mathrm{p} .71-88$. Disponible en: https://doi.org/10.1002/tl.318. 
BROOKS, A.; WARNER, L. y HAMMONS, J. Information literacy leadership: The traits we didn't know we had. College \& Research Libraries News, 2021, vol. 82, nº 6, p. 278. Disponible en: https://doi.org/10.5860/crln.82.6.278.

CAMPAL, F. El bibliotecario docente: ¿formador o aprendiz? [en línea]. Disponible en: $<$ https://www.biblogtecarios.es/felicampal/el-bibliotecario-docente-formador-o-aprendiz/> [Consulta: 5 de mayo de 2021]

CARPENTER, C. y SUTO, M. Qualitative research for occupational and physical therapists. Nueva Jersey: BlackWell Publishing, 2008.

DOMÍNGUEZ AROCA, M.I. La biblioteca y las competencias informacionales en el currículo de los estudiantes de ciencias, medicina y ciencias de la salud de la Universidad de Alcalá. El profesional de la información, 2017, vol. 26, no 3, p. 516-524. Disponible en: https://doi.org/10.3145/epi.2017.may.18.

DORNER, J.L.; TAYLOR, S.E. y HODSON-CARLTON, K. Faculty-librarian collaboration for nursing information literacy: a tiered approach. Reference Services Review, 2001, vol. 29, $\mathrm{n}^{\circ}$ 2, p. 132-141. Disponible en: https://doi.org/10.1108/00907320110394173.

DUCAS, A.; MICHAUD-OYSTRYK, N. y SPEARE, M. Reinventing ourselves: New and emerging roles of academic librarians in Canadian research-intensive universities. College \& Research Libraries, 2020, vol. 81, n 1 , p. 43. Disponible en: https://doi.org/10.5860/crl.81.1.43.

FUNDATOR, R. y MAYBEE, C. Academic librarians as informed learning developers. En K. RANGER (Ed.), Advances in Librarianship: Informed learning applications: Insights from research and practice, vol. 46, p. 81-94. Emerald Publishing Limited, 2019. Disponible en: https://doi.org/10.1108/S0065-283020190000046008.

GARCÍA-MARTÍNEZ, M. Competencia informacional de los estudiantes de enfermería del campus Terres de L'Ebre para buscar y seleccionar información académica en Internet [en línea]. Departamento de Pedagogía, Universidad Rovira y Virgili, Tarragona, 2010. Disponible en: <http:/www.tdx.cat/handle/10803/8952> [Consulta: 5 de junio de 2021]

GARCÍA-MARTÍNEZ, M.; LLEIXÀ FORTUÑO, M.; NIETO DE LA FUENTE, C. y ALBACAR-RIBÓO, N. Competencia informacional en enfermería y otros profesionales de la salud. Index Enfermería, 2011, vol. 20, $\mathrm{n}^{\circ} 4$, p. 257-261. Disponible en: https://dx.doi.org/10.4321/S1132-12962011000300010.

GÓMEZ-HERNÁNDEZ, J.A. Las bibliotecas universitarias y el desarrollo de las competencias informacionales en los profesores y los estudiantes. Revista de Universidad y Sociedad del Conocimiento (RUSC), 2010, vol. 7, n 2, p. 3949. Disponible en: http://dx.doi.org/10.7238/rusc.v7i2.980.

GONZÁLEZ FERNÁNDEZ-VILLAVICENCIO, N. El bibliotecario-docente-universitario es un "sin papeles". Anuario ThinkEPI, 2016, vol. 10, p. 60-67. Disponible en: https://doi.org/10.3145/thinkepi.2016.06.

GONZÁLEZ FERNÁNDEZ-VILLAVICENCIO, N.; BARRERA GÓMEZ, J.A.; SAEN DE CASAS, E. y MOYA OROZCO, V.M. Bibliotecarios e innovación docente: La oportunidad de los planes tutoriales y los trabajos de fin de grado. BiD Textos universitaris de biblioteconomia $i$ documentació, 2016, $\mathrm{n}^{\circ}$ 36. Disponible en: http://dx.doi.org/10.1344/BiD2016.36.14.

GUERRA-MARTIIN, M.D.; LIMA SERRANO, M.; ZAMBRANO DOMÍNGUEZ, E.M. y LIMA- RODRÍGUEZ, J.S. ¿Es efectivo el aprendizaje sobre alfabetización en información para estudiantes de enfermería? Enfermería Global, 2014, vol. 13, nº 4, p. 90-102. Disponible en: https://doi.org/10.6018/eglobal.13.4.182541.

HERNÁNDEZ-HERNÁNDEZ, C.J. Un plan de formación en competencias de información a través de aulas virtuales: análisis de una experiencia con alumnado universitario. Revista de Universidad y Sociedad del Conocimiento (RUSC), 2010, vol. 7, nº 2, p. 50-62. Disponible en: http://dx.doi.org/10.7238/rusc.v7i2.981.

JACOBS, S.K.; ROSENFELD, P. y HABER, J. Information literacy as the foundation for evidence-based practice in graduate nursing education: a curriculum-integrated approach. Journal of Professional Nursing, 2003, vol. 19, $\mathrm{n}^{\circ} 5$, p. 320-328. Disponible en: https://doi.org/10.1016/S8755-7223(03)00097-8.

JACOBSEN, H.E. y ANDENÆS, R. Third year nursing students' understanding of how to find and evaluate information from bibliographic databases and Internet sites. Nurse education today, 2011, vol. 31, $\mathrm{n}^{\circ}$ 8, p. 898-903. Disponible en: https://doi.org/10.1016/j.nedt.2011.01.003.

JANKE, R.; PESUT, B. y ERBACKER, L. Promoting information literacy through collaborative service learning in an undergraduate research course. Nurse education today, 2012, vol. 32, $\mathrm{n}^{\mathrm{o}}$ 8, p. 920-923. Disponible en: https://doi.org/10.1016/j. nedt.2011.09.016.

JULIEN, H. y PECOSKIE, J.J. Librarians' experiences of the teaching role: Grounded in campus relationships. Library \& Information Science Research, 2009, vol. 31, no 3, p. 149-154. Disponible en: https://doi.org/10.29173/cais117.

LIMBERG, L.; ALEXANDERSSON, M. y LANTZ-ANDERSSON, A. What matters? shaping meaningful learning through teaching information. Libri, 2008, $\mathrm{n}^{\circ}$ 58, p. 82-91. Disponible en: https://doi.org/10.1515/libr.2008.010.

LUPTON, M. The getting of wisdom: Reflections of a teaching librarian. Australian Academic \& Research Libraries, 2002, vol. 33, $\mathrm{n}^{\circ}$ 2, p. 75-85. Disponible en: https://doi.org/10.1080/00048623.2002.10755184.

MACKEY, T.P. y JACOBSON, T.E. Information literacy: a collaborative endeavor. College Teaching, 2005, vol. 53, $\mathrm{n}^{\circ} 4$, p. 140-144. Disponible en: https://doi.org/10.3200/ctch.53.4.140-144. 
MANSO PEREA, C; CUEVAS CERVERÓ, A; MARTÍNEZ MIGUEL, E. y GARCÍA-CARPINTERO BLAS, E. Competencias informacionales en ciencias de la salud una propuesta formativa para estudiantes de grado en enfermería. Revista iberoamericana de Ciência da Informação (RICI), 2015, vol. 8, nº 1, p. 1-13. Disponible en: $<$ https://periodicos.unb.br/index.php/RICI/article/view/1878> [Consulta: 10 de mayo de 2021]

MANSO PEREA, C.; CUEVAS CERVERÓ, A. y GONZÁLEZ CERVANTES, S. Competencias informacionales en los estudios de grado en enfermería: el caso español. Revista Española de Documentación Científica, 2019, vol. 42, $\mathrm{n}^{\circ}$ 1, p. e229. Disponible en: https://doi.org/10.3989/redc.2019.1.1578.

ORTOLL ESPINET, E. La competencia informacional en las ciencias de la salud: una visión desde las universidades españolas. Revista Española de Documentación Científica, 2004, vol. 27, nº 2, p. 221-241. Disponible en: https://doi.org/10.3989/redc.2004.v27.i2.153.

ØVERN, K.M. Faculty-library collaboration: two pedagogical approaches. Journal of Information Literacy, 2014 , vol. 8, $\mathrm{n}^{\circ} 2$, p. 36-55. Disponible en: http://dx.doi.org/10.11645/8.2.1910.

ÖZBIÇAKÇI, Ş.; GEZER, N. y BILIK, Ö. Comparison of effects of training programs for final year nursing students in Turkey: Differences in self-efficacy with regard to information literacy. Nurse education today, 2015, vol. 35, $\mathrm{n}^{\mathrm{o}}$ 2. Disponible en: https://doi.org/10.1016/j.nedt.2014.10.008.

PEACOCK, J. Teaching skills for teaching librarians: Postcards from the edge of the educational paradigm. Australian Academic and Research Libraries, 2001, vol. 32, $\mathrm{n}^{\mathrm{0}}$ 1, p. 26-42. Disponible en: https://doi.org/10.1080/00048623.2001.10755141.

POPE, C. y MAYS, N. Qualitative research in health care. $4^{\text {a }}$ ed. Nueva Jersey: Blackwell Publishing, 2019.

REALE, M. The indispensable academic librarian: teaching and collaborating for change. Chicago: American Library Association, 2018.

SERRANO VICENTE, R. Alfabetización en información en bibliotecas universitarias. Algunas experiencias en el ámbito anglosajón. Actas de las $9^{a}$ Jornadas Españolas de Documentación “Infogestión”. p. 509-519. Madrid: FESABID, 2005. Disponible en: < https://dadun.unav.edu/handle/10171/2614> [Consulta: 26 de mayo de 2021]

SIMMONS, M. y CORRALL, S. The changing educational needs of subject librarians: a survey of UK practitioner opinions and course content. Education for Information, 2011, vol. 28, $\mathrm{n}^{\mathrm{o}}$ 1, p. 21-44. Disponible en: https://doi.org/10.3233/efi-2010-0890.

SOBEL, K.; RAMSEY, P. y JONES, G. The professor-librarian: academic librarians teaching credit-bearing courses. Public Services Quarterly, 2018, vol. 14, $\mathrm{n}^{\mathrm{o}}$ 1, p. 1-21. Disponible en: https://doi.org/10.1080/15228959.2017.1342584.

STOMBAUGH, A. et al. Using lesson study to integrate information literacy throughout the curriculum. Nurse educator, 2013, vol. 38, no 4, p. 173-177. Disponible en: https://doi.org/10.1097/nne.0b013e318296db56.

TARRANT, M.; DODGSON, J.E. y LAW, B.V. A curricular approach to improve the information literacy and academic writing skills of part-time post-registration nursing students in Hong Kong. Nurse Education Today, 2008, vol. 28, $\mathrm{n}^{\circ}$ 4, p. 458-468. Disponible en: https://doi.org/10.1016/j. nedt.2007.08.001.

TEJADA ARTIGAS, C. y MARTÍNEZ GONZÁLEZ, B. Perfiles profesionales del Sistema Bibliotecario Español: fichas de caracterización [en línea]. Consejo de Cooperación Bibliotecaria. Grupo de Trabajo de Perfiles Profesionales. 2a ed. Madrid: Ministerio de Educación, Cultura y Deporte, 2019. Disponible en: $<$ https://www.libreria.culturaydeporte.gob.es/libro/perfiles-profesio-nales-del-sistema-bibliotecario-espanol-fichasde-caracterizacion_1325/> [Consulta: 19 de julio de 2021]

TISCAREÑO ARROȲO, M. y CORTÉS VERA, J.D.J. Competencias informacionales de estudiantes universitarios: una responsabilidad compartida. Una revisión de la literatura en países latinoamericanos de habla hispana. Revista Interamericana de Bibliotecología, 2014, vol. 37, $\mathrm{n}^{\circ}$ 2, p. 117-126.

TONG, A.; SAINSBURY, P. y CRAIG, J. Consolidated criteria for reporting qualitative research (COREQ): A 32-item checklist for interviews and focus groups. International Journal for Quality in Heal Care, 2007, vol. 19, n 6, p. 349357. Disponible en: https://doi.org/10.1093/intqhe/mzm042.

VERHEY, M.P. Information literacy in an undergraduate nursing curriculum: development, implementation, and evaluation. Journal of Nursing Education, 1999, vol. 38, n 6, p. 252-259. https://doi.org/10.3928/0148-483419990901-05.

WALLACE, M.C.; SHORTEN, A.; CROOKEs, P.A.; MCGURK, C. y BREWER, C. Integrating information literacies into an undergraduate nursing programme. Nurse Education Today, 1999, vol. 19, n 2, p. 136-141. Disponible en: https://doi.org/10.1054/nedt.1999.0621.

WALTER, S. Librarians as teachers: A qualitative inquiry into professional identity. College \& Research Libraries, 2008, vol. 69, $\mathrm{n}^{\text {o }}$ 1, p. 51-71. Disponible en: https://doi.org/10.5860/crl.69.1.51.

WESTBROCK, T. y FABIAN, S. Proficiencies for instruction librarians: is there still a disconnect between professional education and professional responsibilities? College and Research Libraries, 2010, vol. 71, $\mathrm{n}^{\circ}$ 6, p. 569-590. Disponible en: http://dx.doi.org/10.5860/crl-75r1. 
WHEELER, E. y MCKINNEY, P. Are librarians teachers? Investigating academic librarians' perceptions of their own teaching roles. Journal of Information Literacy, 2015, vol. 9, $\mathrm{n}^{\mathrm{o}}$ 2, p. 111-128. Disponible en: https://doi.org/10.11645/9.2.1985.

WISHKOSKI, R.; LUNDSTROM, K. y DAVIS, E. Librarians in the lead: A case for interdisciplinary faculty collaboration on assignment design. Communications in Information Literacy, 2018, vol. 12, $\mathrm{n}^{\mathrm{o}} 2$, p. $166-192$. Disponible en: https://doi.org/10.15760/comminfolit.2018.12.2.7.

XU, L. y GIL, N. Librarians as co-teachers and curators: integrating information literacy in a studio art course at a liberal arts college. Art Documentation: Journal of the Art Libraries Society of North America, 2017, vol. 36, nº 1, p. 122136. Disponible en: https://doi.org/10.1086/691376. 